\title{
MENTALIZACIÓN COMO HABILIDAD Y CULTURA \\ (Discusión las aportaciones del Prof. Fonagy) $)^{1}$
}

\author{
Carlos Rodríguez Sutil ${ }^{2}$ \\ IPR, Madrid, España
}

La mente no nace con el individuo sino que se desarrolla en el contexto de la interacción humana y no es exclusivamente interna sino también externa, principalmente externa, es decir, el contexto pragmático de las relaciones interpersonales. El espacio interno es algo creado, como podemos ver, por ejemplo, en la descripción proporcionada por Vygotsky (1977) de cómo se forma el lenguaje egocéntrico, como una fase intermedia de "transición" entre el lenguaje externo (social) y el interno, cuando el niño se está hablando a sí mismo, por ejemplo, explicando las acciones de su juego, para nadie, salvo para sí mismo, pero en voz alta. Siempre que entendamos que "tener en mente la mente del paciente" equivale a empatía y reconocimiento, y que la capacidad del paciente para "interpretar la conducta como motivada por estados mentales" puede ser sustituida por la palabra "agencia" nuestras diferencias con la Teoría de la Mentalización podrían ser en gran medida cuestión sólo de lenguaje.

Palabras clave: Mentalización, Lenguaje, Reconocimiento, Agencia

The mind is not born with the individual but develops in the context of human interaction and the mind is not only internal but also external, mainly external, that is, the pragmatic context of interpersonal relationships. The internal space is something that is created, as we can see, for example, in the description supplied by Vygotsky (1977) of how the egocentric speech is built, as an intermediate phase or "transition" between the external language (social) and inner speech, when the child is talking to himself, for example, explaining the actions of his game, for anyone but himself, but aloud. Whenever we understand that "having the patients mind in mind" is equivalent to empathy and recognition, and that the patient's capacity to "interpret behavior as motivated by mental states" could be substituted by "agency", my conclusion is that our differences could be in a great proportion merely question of language.

Key Words: Mentalization, Language, Recognition, Agency

English Title: Mentalizing: Artifice and Culture (A discussion to Prof. Fonagy's Contributions)

\section{Cita bibliográfica / Reference citation:}

Rodríguez Sutil, Carlos. (2016). Mentalización como habilidad y cultura (Discusión de las aportaciones del Prof. Fonagy. Clínica e Investigación Relacional, 10 (3): 649-664. [ISSN 1988-2939] [Recuperado de www.ceir.info ] DOI: 10.21110/19882939.2016.100303

\footnotetext{
1 Leído como contribución a la discusión de las aportaciones del Prof. Peter Fonagy en las IV Jornadas de Psicoanálisis Relacional: MENTALIZACIÓN, Salamanca, 28 y 29 de Octubre de 2016

2 Presidente del Instituto de Psicoterapia Relacional, Madrid, España, Miembro de IARPP. Doctor en Psicología, Psicoanalista y Psicoterapeuta. Dirección de contacto: crsutil56@gmail.com 
Antes de que la práctica hubiera demostrado que las letras del alfabeto podían enlazar aladas palabras en línea tras línea, nadie podría haber imaginado un almacén o una tabla de cera dentro de la mente.

Ivan Illich (1993, pp. 38-39)

Tira el libro. (Leon Hoffman citado por Paul Fonagy, 1999 b)

\section{INTRODUCCIÓN}

Chuang Tsé y Hui Tzu estaban paseando por la ribera del río Hao, cuando Chuang Tsé dijo: - ¿Has visto cómo suben los peces a la superficie y nadan por ella a su placer? Eso es lo que realmente le gusta al pez.

- Tú no eres un pez - replicó Hui Tzú -; por tanto, ¿cómo puedes saber lo que les gusta? Chuang Tsé le dijo:

- Tú no eres yo; por consiguiente, ¿cómo puedes saber que yo no sé lo que le gusta a un pez?

Dijo entonces Hui Tzú:

- Yo no soy tú, por tanto no puedo saber lo que tú sabes. Sin embargo, el hecho definitivo es que tú no eres un pez, y eso demuestra que no puedes saber lo que realmente le gusta a un pez.

Replicó Chuang Tsé:

- Ya. Pero si no te importa, volvamos a retomar la primera cuestión. Tú me preguntaste cómo podía saber yo lo que le gusta a un pez. Por tanto, tú ya sabías que yo lo sabía cuando hiciste la pregunta. Y yo lo sé por el hecho de estar aquí, en la orilla del río Hao.

Este cuento taoista muestra con nitidez las grandes contradicciones y dificultades con Is que nos enfrentamos cuando intentamos teorizar con lo que realmente ocurre dentro de la mente de las personas. Es un terreno lleno de arenas movedizas. Tomemos otra perspectiva sobre este enigma. Vamos a ver un fragmento de un antiguo film dirigido por el gran maestro Alfred Hitchcock "Dial M for Murder", en español "Crimen Perfecto". Leamos primer un breve resumen de la trama.

Tony Wendice está casado con una mujer rica de la alta sociedad, Margot. Ella tiene una aventura con un escritor americano de novelas de crímenes, Mark Halliday. Tony descubre la aventura y decide asesinarla, tanto por venganza como para seguir financiando su confortable estilo de vida con el dinero de ella. Tony se encuentra con un antiguo conocido de la universidad, Swann, que se ha convertido en un criminal de poca monta. Tony ha robado el bolso de Margot que contenía una carta de Mark y la ha estado chantajeando de manera anónima. Después de engañar a Swann para que deje sus huellas digitales en la 
carta, le ofrece una cantidad de dinero para que mate a Margot; si rechaza la oferta, Tony le llevará a la policía como el chantajista de Margot.

El plan es: la noche siguiente Tony llevará a Mark a una fiesta dejando a Margot en casa y escondiendo la llave del apartamento al lado de la puerta de entrada. Swann tendrá que colarse cuando Margot esté dormida y esconderse detrás de las cortinas. A las once Tony llamará a casa desde la fiesta y Swann debe matar a Margot cuando responda al teléfono, abrirá las puertas del jardín, sugiriendo que hubo un robo que salió mal, y saldrá por la puerta delantera, dejando de nuevo la llave. Cuando Margot responde al teléfono, Swann intenta estrangularla pero ella logra alcanzar unas tijeras y lo mata a él. Toma el auricular del teléfono y pide ayuda. Cuando Tony regresa al apartamento llama a la policía, pero antes de que lleguen, cambia la llave que cree que es de Margot del bolsillo de Swann al bolso de ella y pone la carta de Mark en el bolsillo de Swann. El inspector jefe Hubbard arresta a Margot tras llegar a la conclusión de que ha matado a Swann porque la estaba chantajeando. Margot es hallada culpable y condenada a muerte. El día anterior a la ejecución prevista, Hubbard pregunta a Tony por unas grandes cantidades de dinero en metálico que ha estado gastando, le engaña para descubrir que tiene la llave del apartamento guardada en la gabardina y discretamente cambia la gabardina de Tony por la suya. Tan pronto como Tony se va, vuelve a entrar en el apartamento y Mark le sigue. Hubbard ya había descubierto que la llave que Margot tenía en su bolso era la de Swann y dedujo que Swann había vuelto a colocar la llave en su escondite después de abrir la puerta. Tony, al descubrir que no tiene la llave, recupera el bolso de Margot en la comisaría. Pero la llave de Margot no funciona por lo que finalmente utiliza la llave escondida para abrir la puerta, demostrando su culpabilidad. (1:36:46 - 1:37:24 $-1: 41: 16-1: 45: 20)$

Una de las últimas escenas muestra a Tony razonando "internamente" sobre las llaves y a Hubbard ofreciendo a la audiencia la voz en off de lo que está pasando por su cabeza.

00000000000000

Desde la época de Platón (Teeteto) se considera que pensar es hablar con uno mismo, pero no fue hasta Descartes cuando se hizo dominante la concepción del pensamiento como una actividad interna en un espacio interno cerrado, la mente (o el cerebro). Una de las ideas más peligrosas para un filósofo, decía Wittgenstein $(1945-48, \$ 605, \$ 606)$, es que pensamos en nuestras cabezas, en un espacio cerrado, escondido. Lo que vemos en el film de Hitchcock es real, y en cierta medida interno, sin embargo las señales son totalmente exteriores y 
muestran el resultado de un largo proceso evolutivo en un adulto inteligente y sofisticado, aunque moralmente deficiente. Ese espacio "interno" no es algo dado desde el principio.

Relacionales, intersubjetivistas y otros neopsicoanalistas, al igual que Fonagy (2008), en resumen, todos nosotros manifestamos nuestra oposición al dualismo ontológico de Descartes y su postulado de una mente interna, más o menos aislada. Esta ontología se convirtió hace ya mucho tiempo en el lenguaje oficial -la "doctrina oficial" como la llamó Gilbert Ryle (1945)- y hoy en día se ha convertido en un trabajo de Hércules el deshacernos de ella. De acuerdo con esta doctrina todos los seres humanos, excepto los niños y los idiotas, viven dos historias paralelas: la del cuerpo y la de la mente. El alma, o la mente, es la existencia más inmediatamente cognoscible para cada persona. La mente de los otros no se puede conocer directamente, aunque puede ser inferida: de alguna manera es fosforescente. La sede de esta vida interior, el pensamiento, es la cabeza o, más en concreto, el cerebro. Uno de los resultados erróneos de este esquema está en considerar que toda conducta de la persona tiene una causa interior, oculta, en principio desconocida. Pero el futuro también nos es desconocido, como dice Ryle, y eso no quiere decir en ningún modo que esté escondido. El otro error consiste en trasponer la causalidad física a los eventos mentales (Wittgenstein, 1945-49). La doctrina oficial, por tanto, toma como algo dado, primitivo, la existencia de los mecanismos internos.

Creo que los argumentos utilizados por Fonagy a veces caen, sin pretenderlo, en los errores habituales debidos a la perspectiva cartesiana. Por ejemplo, Fonagy plantea su posición anticartesiana de la siguiente manera:

Nuestro enfoque de forma explícita rechaza los supuestos cartesianos clásicos de que los estados mentales son captados mediante introspección; por el contrario, los estados mentales son descubiertos mediante interacciones en espejo contingentes con el cuidador. (2008, p. 10; subrayado nuestro)

En lugar de esto yo diría, probablemente, no que los "estados mentales" son descubiertos sino que son creados a través de la interacción social. La expresión "estados mentales" es un término dependiente de la cultura usado habitualmente en las sociedades occidentales. Existe un principio en el cartesianismo más básico que el de la adquisición de contenidos mediante introspección, y ese es la separación ontológica de las dos sustancias, mente y materia, espíritu y extensión, interno y externo.

La posición que adoptemos respecto al cartesianismo determina en cierta medida nuestra posición en el marco clínico. El psicoanálisis entendido como una técnica asume que una mente aislada, el analista, hace algo a otra mente aislada, el paciente o, en el peor de los casos, a la inversa. El resultado no puede ser un ser humano más sano; el ser humano es 
dejado aparte y el mejor resultado es un objeto más pulido. Las recomendaciones técnicas se convierten en reglas fijas que se mantienen hoy en nuestro "superyó psicoanalítico colectivo" (Orange, Atwood, Stolorow, 1997). Experimentamos nuestra relación con el otro -de acuerdo con la posición técnica- como si las cosas pasaran de manera secuencial, en forma de acción y reacción, como en un puente de dirección única, que da lugar a una causalidad lineal. En resumen, uno es agente y el otro paciente, en algunos casos de forma alternativa, en una especie de relación complementaria en la que subjetividad del otro no es reconocida como tal. El ito de la acción y reacción es uno de los que ha dominado el psicoanálisis clásico así como muchos estudios de la psicología cognitiva actual, deudora de la "metáfora computacional". Dicho punto de vista no fue compartido por los pioneros de la psicología evolutiva, inspirada en alguna forma de constructivismo, ya fuera el "constructivismo biológico" (Piaget y seguidores) o el "constructivismo social (Vygotsky, Bruner).

Una de las principales diferencias entre el psicoanálisis relacional y el clásico consiste en un cada vez mayor proximidad o simetría entre el terapeuta y el paciente, formando ambos una dupla terapéutica. La terapia ya no es algo que el terapeuta hace al paciente sino más bien una tarea común de mutualidad y experiencia recíproca, en un campo coconstruido. Fonagy está bien informado sobre los principios y teorías del psicoanálisis relacional (Cf. Fonagy, 1998). Citémosle a él mismo cuando alude al psicoanálisis relacional:

Sería grosero dedicar demasiado espacio a criticar un "trabajo en desarrollo". Estas ideas están en el proceso de creación y una postura excesivamente crítica sólo puede servir para reprimir un proceso tan crítico. Yo preferiría señalar algunas áreas que me gustaría que exploraran los autores en aras de una mayor exhaustividad. (1998, p. 351)

Aprecio profundamente las teorías de Fonagy como instrumentos útiles en el trabajo clínico con pacientes con trastornos de personalidad. La teoría de la mentalización es útil en la medida en que está de acuerdo con la concepción occidental, compartida por terapeuta y paciente, de qué es la mente y cómo funciona. Sin embargo, por la misma razón, dicha teoría, o para ser más exactos, cierta versión fuerte de la misma que se puede revelar mediante una lectura atenta de los textos de Fonagy, puede merecer cierta crítica. Mi intención es, hasta donde yo conozco, señalar algunas áreas de su pensamiento en aras, no de una mayor exhaustividad, sino de un mejor marco de referencia conceptual en su posición teórica, y de esa forma evitar algunas posibles consecuencias indeseables para el trabajo clínico. Bien entendido, esas consecuencias sólo pueden darse en algunos casos ya que, afortunadamente, la mayoría de los clínicos no siguen de manera ciega los principios 
teóricos que aprendieron y son capaces de "tirar el libro" (Leon Hoffman citado por Paul Fonagy, 1999 a). Kohut (1984) decía que la empatía no era algo que él hubiera inventado sino algo que ya estaban usando psicoanalistas de otras orientaciones (freudianos, kleinianos, etc.).

\section{CRÍTICA EPISTEMOLÓGICA A LA TEORÍA DE LA MENTALIZACIÓN}

Una de las capacidades que definen al ser humano es la de tener en cuenta tanto sus propios estados mentales como los de los otros para comprender y predecir la conducta. En psicología evolutiva esto ha recibido el nombre de "una teoría de la mente" (Cf. Wellman and Liu, 2004). Este concepto sirve para agrupar todas las ideas intuitivas que cada uno tiene sobre el funcionamiento mental y la naturaleza de la experiencia, memoria, creencias, atribuciones, intenciones, emociones y deseos tanto propios como pertenecientes a los otros.

Sin embargo, cuando se producen abusos por parte de los padres, la teoría de la mente del hijo se debilita (Fonagy, 1991, 2001; Fonagy y Target, 1996). Fonagy explica de forma convincente que para el niño ya no es seguro pensar sobre los deseos, porque esto implica observar los deseos del progenitor de hacerle daño. Por eso se inhibe una representación elevada de los eventos mentales, lo que proporciona ciertos beneficios para el individuo, permitiéndole, por decirlo así, dar un rodeo contra un dolor mental intolerable. El niño busca la comodidad en una fusión con el objeto, con un 'progenitor rescatador' en la fantasía. Por tanto es lógico concluir que el que el analista piense y hable sobre el pensamiento del paciente puede ayudar a reparar los defectos globales o focales en la capacidad mentalizadora del paciente.

Fonagy sugiere que la mentalización es una teoría que ofrece un marco de referencia integrador que puede integrar mente y cerebro y servir como "lenguaje común" para una variedad de modalidades terapéuticas (Fonagy and Allison, 2014, p.373). La mentalización de los pacientes puede ser un factor común a través de varias psicoterapias "no porque los pacientes necesiten aprender sobre los contenidos de sus mentes o de las mentes de otros, sino porque la mentalización puede ser una forma genérica de incrementar la confianza epistémica [confiar en la realidad de lo que dice el terapeuta] y lograr as'un cambio en el funcionamiento mental" (Fonagy and Allison, 2014, p.477).

Teniendo todo esto en cuenta parece conveniente buscar una definición precisa de "mentalización": 
Definimos la mentalización como una forma de actividad imaginativa mental principalmente preconsciente, digamos, de interpretar la conducta humana en términos de estados mentales intencionales (p.e. necesidades, deseos, sentimientos, creencias, objetivos, propósitos y razones). La mentalización es imaginativa porque tenemos que imaginar lo que las otras personas podrían estar pensando o sintiendo; un importante indicador de una mentalización de alta calidad es la conciencia de que no sabemos ni podemos saber de forma plena qué es lo que está en la mente del otro. Sugerimos que un tipo semejante de salto imaginativo es necesario para comprender la propia experiencia mental, especialmente en relación con temas emocionalmente cargados. Para concebir que los otros tienen mente, el individuo necesita un sistema de representación simbólica para los estados mentales y también debe ser capaz de activar selectivamente estados mentales en línea con intenciones particulares, que requieren control de la atención. (Fonagy, 2008, p. 4, énfasis añadido)

Ruego disculpas a Fonagy si compare su posición -compleja y comprensiva- en algunos aspectos con la de Andrew Meltzoff. A mediados de los años setenta, Meltzoff descubrió que los bebés de entre 12 y 21 días pueden imitar tanto gestos faciales como los manuales (Meltzoff and Moore, 1977). Esta conducta implica que los humanos recién nacidos equiparan sus propias conductas no vistas con los gestos que ven realizar a los otros. Pero más importante para lo que aquí nos ocupa. Meltzoff (2007) propuso la hipótesis "como yo" para el desarrollo infantil: 'aquí hay algo que es como yo'. El bebé experimenta regularmente una asociación entre sus propios actos y los estados mentales subyacentes. Posteriormente proyecta sus experiencias internas a los otros que realizan actos similares, y comienza a adquirir una comprensión de las "otras mentes": sus estados mentales, emociones, deseos, etcétera. La imitación es una capacidad innata y la comprensión de los estados mentales de los otros es un derivado. De una forma semejante, Fonagy sugiere: "... la capacidad de dar significado a las experiencias psicológicas evoluciona como resultado de nuestro descubrimiento de la mente detrás de las acciones de los otros, que se desarrolla de manera óptima en un contexto social seguro y a salvo" (2008, p. 29, énfasis añadido). Yo diría no que se "desarrolla" sino que se "aprende" mediante las instrucciones del cuidador.

La hipótesis de Meltzoff (y Fonagy) es deudora del argumento per analogiam propuesto por el filósofo ingés John Stuart Mill, en el siglo XIX, para resolver el problema de las "otras mentes" y los riesgos del solipsismo y de la mente aislada derivados del cartesianismo. De acuerdo con esto, la afirmación de que las otras personas también tienen una conciencia es una conclusión que derivamos de sus acciones y manifestaciones visibles, 
con el objetivo de hacer comprensible su conducta. Lo que quiere decir que atribuir conciencia a los otros es una inferencia y no una experiencia irrefutable.

Yo sugeriría que el bebé no compara un estado interno con una simulación visual, sino simplemente que estamos "programados" (es nuestra naturaleza) de una forma que nos permite desplegar de forma espontánea una una respuesta emocional, por ejemplo, frente a una sonrisa del cuidador, y de esa forma compartimos su "estado mental". El "estado mental" no es en principio una propiedad individual sino que es compartido por al menos dos personas (CF. Knoblauch, 2000, p. 158); la mente inconsciente también es compartida por dos o más personas (Lyons-Ruth, 1999, Gerson, 2004). Cuando pensamos de forma permanente que en realidad son entidades privadas, se sigue que son expresión de sentimientos internos. No obstante, aun reconociendo que las emociones requieren procesamiento cognitivo y respuestas fisiológicas, algo que no me molesta aceptar, se trata de fenómenos sociales que a menudo ocurren por contagio perceptivo, sin ninguna mediación cognitiva.

Fonagy postula que la representación simbólica de los estados mentales puede ser vista como un prerrequisito para el sentido de identidad (Fonagy and Target, 1996; Target and Fonagy, 1996; Fonagy and Allison, 2014). Por ello, los pacientes con trastornos graves de la personalidad inhiben su función reflexiva, y tienen un acceso limitado a su propio mundo representacional. Pero yo sospecho que lo que tienen estos pacientes no es un mundo representacional oculto o inhibido, sino que más bien sufren de una carencia, es decir, muestran déficit en su mundo representacional. No han adquirido las habilidades necesarias para representar y en consecuencia no tienen un mundo representacional "completo".

Es usual que construyamos nuestras teorías psicológicas (cognitivas) en base a representaciones mentales. Sin embargo, todavía no sabemos cómo representa la representación. Me siento incómodo con el lenguaje de la psicología cognitiva que impregna al psicoanálisis relacional, y no solo relacional. Quizá el enfoque mentalista sería adecuado para todos los pacientes, independientemente de sus trastornos. Y quizá los pacientes con un trastorno límite se beneficiarían de una actitud mentalizadora por parte de sus terapeutas. Pero imagino que estos pacientes tienen una mayor necesidad de una aceptación y un reconocimiento empáticos, y sólo de forma secundaria de una actitud mentalizadora. La respuesta de Fonagy probablemente es que la mentalización no es incompatible en absoluto con la aceptación y el reconocimiento, pero quizá sería mejor pensar en términos de patrones relacionales - desde un punto de vista externo y descriptivoy en la ritmicidad (concepto escasamente mentalista) en la relación terapeuta-paciente. 
En otro lugar Fonagy (Fonagy, 1999 a; Fonagy, Steele, Steele, Leigh, Kennedy, Mattoon, and Target, 1995) confirma la idea de que las relaciones -la percepción de un analista que tiene empatía o intenciones sanadoras, y no solo interpretaciones- lleva a cambios en las estructuras representacionales. "Por tanto no hay diferencia cualitativa entre los medios con los que se logra el cambio terapéutico, mediante la interpretación o mediante una nueva relación" (Fonagy, 1999 a). Percibir al terapeuta como alguien que escucha con empatía o tiene intenciones sanadoras trae cambios mediante el mismo mecanismo que las interpretaciones.

Pero estas dos posturas "técnicas" no son equivalentes, pues si nuestra praxis se basa principalmente en las interpretaciones esto quiere decir, de manera encubierta, que estamos en posesión de la verdad sobre los problemas del paciente, y en consecuencia adoptamos la posición clásica analista-paciente. Recientemente Margy Sperry (2013) basó su crítica a las ideas de Fonagy en un fundamento semejante. Cuando Fonagy ve al paciente bloqueado en un estadio prementalizador del desarrollo, Sperry dice:

Dicho supuesto coloca al analista como un intérprete autorizado y objetivo de los significados y de las fuentes de los procesos mentalizadores del paciente, y minimiza las formas en que ese analista puede contribuir al propio fenómeno que está explicando. (id., p. 686).

Pero Fonagy seguramente respondería que:

Sin embargo, la interpretación no aparece forzada de cierta forma dictatorial sino que es ofrecida como un comienzo para dar sentido a lo que en otro caso puede ser un acontecimiento o un sentimiento sin significado. Se convierte en un camino por el que el terapeuta puede demostrar que hay pensamiento en su propia mente sobre la mente del paciente e inevitablemente puede ser dada con diferentes grados de competencia y sensibilidad. (Bateman y Fonagy, 2004, p. 131)

Sin embargo, es muy probable que en un análisis relacional utilicemos la interpretación en una menor extensión que en un análisis clásico, y menos todavía con pacientes con un funcionamiento límite.

Fonagy (2008, p. 4) dice que la experiencia del bebé de sí mismo como teniendo una mente o un self no es algo genéticamente dado, sino que evoluciona desde la primera infancia a través de la niñez y que depende de la interacción con cuidadores en sintonía. Pero al mismo tiempo no puedo estar más en desacuerdo con lo que parece ser su darwinismo social. Fonagy (2008, pp. 5-9) afirma que la mentalización es la "cumbre evolutiva" del logro intelectual humano, después de dos millones de años de procesos selectivos en la evolución 
humana -una propuesta que eliminando las referencias biológicas seguramente Hegel habría suscrito-. Esto implica un grave riesgo de mala interpretación al identificar el cambio cultural con la evolución darviniana. Cuando nos referimos a la historia de la humanidad la prudencia nos recomienda pensar simplemente en un sentido estrictamente no evaluativo, en lugar de postular la existencia de una evolución positiva, evitando toda idea de perfeccionamiento y el culturocentrismo. Parece ser que inspirado en el darvinismo social, Fonagy sugiere que nuestra excepcional inteligencia evolucionó no sólo para enfrentarse con las fuerzas hostiles de la naturaleza sino también contra la competencia de otras gentes. Este hecho no se basa en la genética; el "cerebro social" debe alcanzar cada vez mayores niveles de sofisticación para "seguir arriba". Sospecho, sin embargo, que esta competitividad sólo es consistente con los valores occidentales.

La mentalización implica dotar de sentido a las acciones propias y de los otros en base a los estados mentales intencionales (IIF) (Fonagy, 2008), es decir, tratar al objeto cuya conducta quieres predecir como un agente racional o intencional con creencias y deseos. Durante el segundo año, los niños comprenden que tanto ellos como los otros son agentes intencionales cuyas acciones están causadas por estados mentales previos (deseos, intenciones) y que sus acciones pueden producir cambios en las mentes así como en los cuerpos (id. p. 26). En relación con esto se podría responder que en muchas ocasiones el deseo y la intención no precede a la acción sino que la acompaña o, quizá deberíamos decir, que cada acto es una expresión del deseo o es una acción intencional. Quizá Fonagy transmitía aquí una imagen excesivamente introspectiva de la dinámica acción-reacción que se establece entre el infante y su entorno social. Seguramente se trata de un fenómeno mucho más complejo: ¿De quién es el deseo?¿Cuándo aparece exactamente el deseo?¿Yo tengo un deseo o el deseo me tiene a mí? Las explicaciones de Fonagy tienen un sonido excesivamente representacional o cognitivo. Prefiero no hablar de representaciones sino de patrones de acción, en su mayor medida procedimentales.

Pongamos otro ejemplo. Fonagy añade:

En resumen, la capacidad de dar sentido a las experiencias psicológicas evoluciona como resultado de nuestro descubrimiento de la mente detrás de las acciones de otros, que se desarrolla de manera óptima en un contexto social relativamente a salvo y seguro. (2008, p. 29, énfasis añadido).

Este fragmento suena realmente paradójico. Si tengo que buscar el sentido "detrás" de las acciones del otro esto es porque el contexto social no es en absoluto salvo y seguro. Lo que me hace desconfiar de las buenas intenciones de los otros es su comportamiento a largo plazo, nada interno. Puede haber algunos signos en el aquí y ahora que me hagan 
desconfiar de su "intención". E "intención" es un término generalizado que he aprendido para calificar secuencias largas de conducta. Hay un mntón de cosas que ignoramos, pero la verdad no tiene por qué estar escondida detrás. No hay nada detrás o "nada está oculto" (Malcolm, 1986).

La adquisición de la mentalización -sugiere Fonagy (id. pp. 8-9)- capacita al niño para distinguir la realidad interna de la externa y los procesos internos mentales y emocionales de los acontecimientos interpersonales. En relación con eso debemos objetar que los acontecimientos mentales también son interpersonales por definición: pensar es hablar con uno mismo (Platón), y nos resulta imposible sentir una emoción que no esté situada en el nivel interpersonal. Es un hecho innegable lo que dice Fonagy: la autoconciencia nos permite modificar la forma en que nos presentamos ante los otros e inducirlos a error, "abre la puerta al engaño malicioso" (id., p. 29). Los niños de menos de cuatro años generalmente creen que lo que ellos conocen es conocido por todo el mundo. Los niños de menos de cuatro años normalmente creen que lo que ellos saben lo sabe todo el mundo. Los experimentos relacionados con la tarea de la falsa creencia parecen poseer un valor probatorio, aunque algunos investigadores muestran desacuerdo (Cf. Bloom and German, 2000). En cualquier caso, la mentira existe, pero es una habilidad que debemos aprender (Wittgenstein: la mentira es un juego del lenguaje que necesita ser aprendido como cualquier otro 1945-49, \$ 249). Y sólo es posible dudar cuando existe la certeza. Podemos afirmar que sólo es posible mentir cuando existe la verdad. Eso no quiere decir que todas nuestras relaciones sociales estén basadas en el engaño. Para la mayoría de nuestras acciones sólo tenemos que tener en cuenta su sentido "aparente" debido a que la mayor parte de nuestra vida social está basada en la confianza.

El filósofo francés e historiador de las ideas Michel Foucault (1988) introdujo el término "tecnologías del yo" que posiblemente es aplicable a la teoría de la mentalización. Hay cuatro tipos principales de "tecnologías":

(1) Las tecnologías de producción;

(2) Las tecnologías de sistemas de signos;

(3) Las tecnologías de poder, que determinan las conductas de los individuos y los remiten a ciertos fines de dominación, a una objetivización del sujeto; y

(4) Las tecnologías del yo,

Cada una de ellas implica ciertos modos de entrenamiento y modificación de los individuos, dependen del momento histórico, y no sólo implican la adquisición de ciertas capacidades sino también la adquisición de ciertas actitudes. Las tecnologías del yo 
permiten a los individuos realizar una cierta cantidad de operaciones en sus cuerpos y almas, pensamientos, conducta y modos de ser, transformándose así a sí mismos para alcanzar cierto estado de felicidad, pureza, sabiduría, perfección o inmortalidad.

La consciencia moderna se dedica a dos problemas esenciales que no estaban presentes en la mente de las antiguas culturas: la obediencia a la ley y el autoconocimiento (Foucault, 2001, p. 305). Los dos mandatos de la antigüedad griega eran "cuida de ti mismo" y "conócete a ti mismo" y la segunda ha cubierto y obscurecido a la primera. Dice Bruno Snell (2007, pp. 289-90) que la mala conciencia es un estado mental que no apareció reseñado hasta Eurípides, así como la vergüenza, el embarazo ante los otros. Los héroes homéricos carecían de mala conciencia, por no hablar de la culpa. En lugar de ello tenían las Furias o las Euménides, diosas de la venganza que perseguían a aquellos que habían cometido crímenes de sangre, sobre todo parricidio. En cualquier caso, se trataba de un factor externo a la mente del individuo. No me atrevería a decir que la cultura de la antigua Grecia era inferior a la nuestra.

Por otra parte, los pacientes con trastornos límite a menudo son muy sensibles a los estados mentales del otro, bien comprendidos con objeto de controlarlos y manipularlos (Cf. Fonagy, 1999 b). La mentalización es una tecnología del yo adquirida durante el desarrollo que permite propósitos positivos o negativos. Fonagy nos previene de identificar una mayor mentalización con la major disposición para servir fines prosociales (2008, p. 29). Yo lo pondría en términos piagetianos: superar el estadio de las operaciones concretas hasta alcanzar el estadio de las operaciones formales no significa que se haya alcanzado una moral autónoma. Parece necesario completar la teoría de la mentalización con una teoría del desarrollo moral. Yo recomienddo el esquema tripartito de los estadios del desarrollo moral establecidos por Lawrence Kohlberg (1964; Kohlberg, Levine, and Hewer, 1984): la moral pre-convencional, convencional y post-convencional. La moral post-convencional es un logro alcanzado por algunos individuos, sin relación con su personalidad dominante, aunque está vedada para narcisistas de "piel dura" y sujetos antisociales. En el proceso de toma de decisiones del individuo surgen tres expresiones diferentes que representan tres grupos o tipos de sujetos: "yo lo quiero", "el grupo lo aprueba", y "es lo correcto". El segundo, que se corresponde con la moral convencional, es característico de las personalidades límite, en las que la incorporación "interna" de las reglas todavía no ha sido lograda, mientras que la moral pre-convencional (yo lo quiero) es típica de psicópatas y narcisistas de "piel dura". La moral convencional implica el temor a perder el aprecio del otro significativo.

\section{CONCLUSIONES}


Freud a veces manejaba la concepción de un sujeto inconsciente relativamente aislado de su entorno, un yo solipsista bajo el control del narcisismo primario. Fonagy no mantiene, afortunadamente, conceptos clásicos como el de "narcisismo primario" o la teoría pulsión /instinto, pero queda atrapado si no en el pensamiento cartesiano al menos en alguna forma del lenguaje de la "doctrina oficial". Por ejemplo cuando leemos: "Los niños [pequeños] no saben del todo que ellos están separados, que su mundo interno es algo privado e individual, del cual finalmente se apropiarán o al menos reivindicarán un acceso privilegiado." (Fonagy, 2008, id. p. 31)

La mente no nace con el individuo sino que se desarrolla en el contexto de la interacción humana y no es exclusivamente interna sino también externa, principalmente externa, es decir, el contexto pragmático de las relaciones interpersonales. El espacio interno es algo creado, como podemos ver, por ejemplo, en la descripción proporcionada por Vygotsky (1977) de cómo se forma el lenguaje egocéntrico, como una fase intermedia de "transición" entre el lenguaje externo (social) y el interno, cuando el niño se está hablando a sí mismo, por ejemplo, explicando las acciones de su juego, para nadie, salvo para sí mismo, pero en voz alta.

Morris Eagle (2011, p. 170) decía, hace no mucho, que en la literature psicoanalítica actual las representaciones son inconscientes no sólo porque se trate de procesos defensivos sino porque han sido adquiridas de forma no verbal en los primeros estadios de la vida. Se está refiriendo al inconsciente procedimental (Cf. Lyons-Ruth, 1999). Sin embargo, cuando añade, correctamente, que estas representaciones son semejantes a los hábitos y a las habilidades motóricas incorporadas en el cuerpo, ¿por qué habremos de seguir manteniendo que son "representaciones"? Los seres humanos, como cualquier organismo, se comportan siguiendo habitualmente una secuencia de actos, esa secuencia puede ser representada mediante un esquema o guión, el guión es una reconstrucción que hacemos nosotros mismos y que no tiene por qué estar representada de ninguna forma dentro del cuerpo o del cerebro.

Como afirma Knoblauch (2000, p. 158), los afectos no están en la persona sino que se construyen continuamente como un campo emocional que se desliza entre las personas que se están influyendo. Lo que la persona actúa en la práctica son esquemas operacionales, aprendidos en el contexto, y no imágenes internas.

Lo que ocurre en el interior sólo tiene sentido en el flujo de la vida. (Auch was im Innern vorghet hat nur im Fluss des Lebens Bedeutung) (Wittgenstein, 1951, II, p.30). 
Los errores surgen de dar un valor per se a esas imágenes internas, cuando de hecho la imagen interna solo posee estabilidad si se la contrasta con el uso (Wittgenstein 1945-48, $\int 258, \int 293$, II, p.196/451). El que se queda ciego, pasado un tiempo, pierde la capacidad de representarse el mundo en imágenes visuales. Para Wittgenstein el postulado esencial no son los sistemas representacionales sino la comunicación interpersonal.

No me cuesta reconocer que la teoría de la mentalización proporciona un marco integrativo que podría constituirse en el "lenguaje común" que Fonagy y Allison (2014, p. 375) sugieren. Ellos proponen la mentalización; otros expertos destacan otros conceptos: apego, empatía, reconocimiento, etc. En cualquier caso Fonagy y Allison tienen razón cuando afirman que uno de los factores principales es:

... la experiencia del paciente de otra persona teniendo en su mente la mente del paciente, y que la terapia (...) funciona reviviendo la capacidad del paciente para interpretar que la conducta está motivada por estados mentales, tanto en sí mismos como en los otros. (id.) (subrayado nuestro)

Siempre que entendamos que "tener en mente la mente del paciente" equivale a empatía y reconocimiento, y que la capacidad del paciente para "interpretar la conducta como motivada por estados mentales" puede ser sustituida por la palabra "agencia". Entonces mi conclusión será que nuestras diferencias podrían ser en gran medida cuestión sólo de lenguaje, una confusión de lenguas. Pero no obstante temo que algunas de las expresiones encontradas en los textos de Fonagy pueden animar a algún terapeuta novato a asumir una actitud autoritaria o dogmática.

Algún día deberemos dedicar un tiempo no breve a resolver el asunto de la representación, y su papel central en la psicología contemporánea, no sólo en sus versiones cognitivistas.

\section{REFERENCIAS}

Bateman, A. and Fonagy, P. (2004). Psychotherapy for Borderline Personality Disorders. Mentalizationbased treatment. Oxford: Oxford University Press.

Bloom, P. and German, T.P. (2000). Two reasons to abandon the false belief task as a test of theory of mind. Cognition, 77, B25-B31.

Cavell, M. (2006). Becoming a Subject. Reflections in Philosophy and Psychoanalysis. Nueva York: Oxford University Press.

Eagle, M.N. (2011). From Classical to Contemporary Psychoanalysis. A Critic and Integration. Nueva York: Routledge.

Fonagy, P. (1991). Thinking about Thinking: Some Clinical and Theoretical Considerations in the Treatment of a Borderline Patient. International Journal of Psychoanalysis, 72, 639-656. 
Fonagy, P. (1998 a). Moments of change in psychoanalytic theory: Discussion of a new theory of psychic change. Infant Mental Health Journal, 19, 3, 346-353.

Fonagy, P. (1999 a). The Process of Change and the Change of Processes: What can Change in a 'Good' Analysis. Keynote Address to the Sprig Meeting of Division 39 of the American Psychological Association, New York. En: http://mentalizacion.com.ar/images/notas/The\%2oprocess\%20of\%20change\%20and\%20the\%2ochange\%200f\%20processes.htm.

Fonagy, P. (1999 b). Pathological attachments and therapeutic action. Paper presented to the Developmental and Psychoanalytic Discussion Group, American Psychological Association Meeting, Washington DC, May 13, 1999. PsycheMatters publication [On Line] Available: www.psychematters.com/papers/fonagyzhtm.

Fonagy, P. (2000). Attachment and Borderline Personality Disorder. Journal of the American Psychoanalytic Association, 48, 4, 1129-1146.

Fonagy, P. (2001 a). The Psychoanalysis of Violence. Paper presented to the Dallas Society for Psychoanalytic Psychotherapy March 15, 2001.

Fonagy, P. (2008). The Mentalization-Focused Approach to Social Development. Chapter 1 of Frederic N. Busch (ed.) "Mentalization. Theoretical Considerations, Research Findings, and Clinical Implications." New York: The Analytic Press.

Fonagy, P. and Allison, E. (2014). The role of mentalizing and epistemic trust in the therapeutic relationship. Psychotherapy, 51, 3, 372-380.

Fonagy, P., Steele, M., Steele, H., Leigh, T., Kennedy, R., Mattoon, G. y Target, M. (1995). Attachment, the reflective self, and borderline states: The predictive specificity of the Adult Attachment Interview and pathological emotional development. En S. Goldberg, R. Muir y J. Kerr (comp.) Attachment Theory: Social, developmental, and clinical perspectives. Hillsdale, New Jersey: Analytic Press.

Fonagy, P. y Target, M. (1996). Playing with reality I. International Journal of Psychoanalysis, 77, 217-33.

Foucault, M. (1988). "Technologies of the Self. A Seminar with Michell Foucault", edited by Luther H. Martin, Huck Gutman and Patrick H. Hutton, pp. 16-49. Univ. of Massachusets Press, 1988.

Foucault, M. (2001). L'Hermenéneutique du Sujet. Cours au Collège de France. 1981-1982. Paris: Gallimard/Seuil.

Gerson, S. (2004). The relational unconscious: A core element of intersubjectivity, thirdness, and clinical process. Psychoanalytic Quarterly, LXXIII, 63-98.

Illich, I. (1993). In the Vineyard of the Text: A Commentary to Hugh's Didascalicon. Chicago: The University of Chicago Press. [En el Viñedo del Texto. Etología de la lectura: un comentario al "Didascalicon" de Hugo de San Víctor. México: Fondo de Cultura Económica, 2002.

Knoblauch, S.H. (2000). The Musical Edge of Therapeutic Dialogue. Hillsdale, NJ: The Analytic Press.

Kohut, H. (1984), How Does Analysis Cure? Chicago: University of Chicago Press. Traducción castellana ¿Cómo Cura el Análisis? Buenos Aires: Paidós, 1986.

Kohlberg, L. (1964). The development of moral character. En M.CC. Hoffmann (comp.) Child Development. Nueva York: SAGE. 
Kohlberg, L.; Levine, C. y Hewer, A. (1984). Moral stages: a current formulation and a response to critics. Basilea: Karger.

Lyons-Ruth, K. (1999). The Two-Person Unconscious: Intersubjective Dialogue, Enactive Relational Representation, and the Emergence of New Forms of Relational Organization. Psychoanalytic Inquiry, 19, 576-617

Malcolm, N. (1986). Nothing is Hidden: Wittgenstein's Criticism of his Early Thought. Oxford: Blackwell.

Meltzoff, A. N. (2007). The 'like me' framework for recognizing and becoming an intentional agent. Acta Psychologica, 124, 26-43.

Meltzoff, A.N. and Moore, M.K. (1977). Imitation of Facial and Manual Gestures by Human Neonates. Science, 198, 75-78.

Orange, D. M., Atwood, G. E., Stolorow, R. D. (1997). Working Intersubjectively. Contextualism in Psychoanalytic Practice. Hillsdale: The Analytic Press.

Ryle, G. (1949). The Concept of Mind. Nueva York: Barnes \& Noble.

Snell, B. (2007). El Descubrimiento del Espíritu. Estudios sobre la génesis del pensamiento europeo en los griegos. Barcelona: Acantilado.

Sperry, M. (2013). Putting our heads together: Mentalizing systems. Psychoanalytic Dialogues, 23, 6, 683-699.

Stolorow, R.D., Orange, D.M., y Atwood, G.E. (2001). Cartesian and post-Cartesian trends in relational psychoanalysis. Psychoanalytic Psychology, 18, 468-484.

Target, M. y Fonagy, P. (1996). Playing with reality: 2. The development of psychic reality from a theoretical perspective. International Journal of Psycho-Analysis, 77, 459-479.

Wellman, H. M., \& Liu, D. (2004). Scaling of theory of mind tasks. Child Development, 75, 2, 523-541.

Wittgenstein, L. (1945-48). Zettel (Z). Traducción inglesa de G.E.M. Anscombe; Oxford: Basil Blackwell, 1981.

Wittgenstein, L. (1945-49). Philosophical Investigations. Traducción inglesa de G.E.M. Anscombe; Oxford: Basil Blackwell, 1984. Revised $4^{\text {th }}$ edition by P.M.S. Hacker and Joachim Schulte. Oxford: Wiley-Blackwell, 2009: The German text, with an English translation. Edición bilingüe alemán-español de Alfonso García Suárez y Ulises Moulines "Investigaciones Filosóficas"; Barcelona: Crítica, 1988.

Wittgenstein, L. (1951). Last Writings on the Philosophy of Psychology . Volúmenes I (1948-1949) y II (1949-1951). Ediciones bilingües alemán inglés, traducciones de C.G. Luckardt y M.A.E. Aue, G.H. von Wright y H. Nyman (eds.). Oxford: Basil Blackwell.

Vygotsky, L.S. (1977). Pensamiento y Lenguaje. Buenos Aires: La Pléyade.

Vygotsky, L.S. (1989). Concrete Human Psychology. Soviet Psychology, 27, 53-77.

Original recibido con fecha: $\quad 30-8-2016 \quad$ Revisado: 29-10-2016 Aceptado: 31-10-2016 